\title{
Helical Tubes of FtsZ from Methanococcus jannaschii
}

\author{
J an Löwe* and Linda A. Amos \\ MRC Laboratory of Molecular B iology, Hills Road, \\ Cambridge CB2 2QH, UK \\ *Corresponding author
}

Bacterial cell division depends on the formation of a cytokinetic ring structure, the Z-ring. The bacterial tubulin homologue FtsZ is required for Z-ring formation. FtsZ assembles into various polymeric forms in vitro, indicating a structural role in the septum of bacteria. We have used recombinant FtsZ1 protein from $M$. jannaschii to produce helical tubes and sheets with high yield using the GTP analogue GM PCPP [guanylyl$(\alpha, \beta)$-methylene-diphosphate]. The sheets appear identical to the previously reported $\mathrm{Ca}^{++}$-induced sheets of FtsZ from $M$. jannaschii that were shown to consist of 'thick'-filaments in which two protofilaments run in parallel. Tubes assembled either in $\mathrm{Ca}^{++}$ or in GM PC PP contain filaments whose dimensions indicate that they could be equivalent to the 'thick'-filaments in sheets. Some tubes are hollow but others are filled by additional protein density. Helical FtsZ tubes differ from eukaryotic microtubules in that the filaments curve around the filament axis with a pitch of $\sim 430 \AA$ for $\mathrm{Ca}^{++}$-induced tubes or $590-620 \AA$ for GMPCPP. However, their assembly in vitro as wellordered polymers over distances comparable to the inner circumference of a bacterium may indic ate a role in vivo. Their size and stability make them suitable for use in motility assays.

Key words: Helical reconstruction / Microtubules / Tubulin.

\section{Introduction}

For a bacterium to divide the cell envelope must be moved inwards to yield two daughter cells, a process called septation (review: Donachie, 1993). For septation to proceed, a number of proteins assemble at the site of septum formation in a specific sequence (recent reviews: Bramhill, 1997; Lutkenhaus and Addinall, 1997; Rothfield et al., 1999). After the site for the septum has been selected using the MinCDE system, Fts Z is the first protein known to appear at the septum. FtsZ then directs all other known septum proteins including ZipA, FtsA, FtsQ, FtsW, Ftsl, FtsL, FtsK, and possibly others. Fts Z assembles into a ring structure in vivo as seen by light microscopy of cells and assembles into filaments in vitro, indicating a structural role for FtsZ in cell division. Further evidence for a structural role comes from the fact that $F t s Z$ can be regarded as the bacterial homologue of the eukaryotic cytoskeletal protein tubulin (Erickson, 1995). Tubulin and FtsZ share a common three-dimensional structure (Löwe and Amos, 1998; Nogales et al., 1998b) and form protofilaments in which the longitudinal contact enhances GTP hydrolysis, thus providing a mechanism for polymerisation dependent GTPase regulation (Erickson, 1998; Nogales et al., 1998a). A 6-residue insertion in the $\beta$-strand preceding loop T2 in FtsZ (Nogales et al., 1998a) produces a phenotype that shows FtsZ spirals instead of closed rings inside cells, indicating that Fts $Z$ acts as a structural protein directly guiding the formation of the septum ( $\mathrm{B}$ i and Lutkenhaus, 1992; Addinall and Lutkenhaus, 1996). The homology to tubulin initiated a search for motor proteins in prokaryotes that move on FtsZ tracks. No obvious candidate has been found so far. A major problem in the field arises from the fact that no ultrastructural information about the in vivo structure of the FtsZ polymer is available. This is due to the small size of the septum and the high protein density near the septum. In the meantime, in vitro experiments have found a number of FtsZ polymers (Bramhill and Thompson, 1994; Mukherjee and Lutkenhaus, 1994; Erickson, 1995; Erickson et al., 1996; Yu and Margolin, 1997; Lu et al., 1998; Mukherjee and Lutkenhaus, 1998; Trusca et al., 1998; Löwe and Amos, 1999) that may be related to the in vivo polymer, although it should be kept in mind that in vivo FtsZ polymerisation may be guided by additional factors. Single, curved filaments have been obtained by polymerising Fts Z in solution in the presence of GTP (Lu et al., 1998, 2000; M ukherjee and Lutkenhaus, 1998). When performed on a lipid monolayer, FtsZ polymerisation yielded minirings (Erickson et al., 1996). Sheets of laterally aligned filaments have been obtained under many conditions: in the presence of $\mathrm{Ca}^{++}$(Yu and Margolin, 1997; Löwe and Amos, 1999), DEAE dextran (Erickson etal., 1996), and GTP alone. A low resolution three-dimensional reconstruction of $\mathrm{Ca}^{++}$-induced $M$. jannaschii FtsZ sheets revealed that they are composed of antiparallel double protofilaments (Löwe and Amos, 1999). These FtsZ protofilaments, with a longitudinal spacing of approximately $43 \AA$, could be very well fitted with an atomic model of the FtsZ protofilament derived from the X-ray structure of FtsZ (Löwe and Amos, 1998) and the tubulin protofilament (Nogales et al., 1998b). If only GTP is added, FtsZ assembles mostly into a network of straight filaments that are not well aligned and the filament bundles vary in thickness (Bramhill and Thompson, 1994; Mukherjee and Lutkenhaus, 1994; Erickson, 1995). None of these three classes of FtsZ polymers is well suited for the task FtsZ faces inside the cell. It has been estimated that there are around $15000 \mathrm{FtsZ}$ molecules per E. coli cell (Dai and Lutkenhaus, 1992; Lu et al., 1998). If a continuous ring assembles around the septum 
FtsZ must form a fairly uniform polymer. Single filaments appear to be too short and have a radius of curvature (Erickson and Stoffler, 1996; Erickson et al., 1996) incompatible with the inner cell surface. Furthermore, if the bending of protofilaments would generate force (Lu et al., 2000), each subunit contact in the protofilament would have to sustain the overall force applied to the membrane, which seems unlikely. Sheets are not well suited because there is no natural boundary for growth in the lateral direction. Poorly aligned bundles of protofilaments lack the required order and tend to form a network that is incompatible with ring formation. During our efforts to obtain $\mathrm{Ca}^{++}$. induced sheets of M.jannaschii FtsZ (Löwe and Amos, 1999), most of the polymers formed appeared to be helical tubes but precipitated into a tight mass. We obtained a few pictures of free helical tubes but were unable to produce more. Two recent studies (Trusca et al., 1998; Lu et al., 2000) reported similar tubes of E. coli FtsZ obtained with GTP or GDP and the addition of DEAE dextran, confirming earlier reports of the existence of helical FtsZ tubes (Bramhill and Thompson, 1994; Mukherjee and Lutken- haus, 1994; Erickson, 1995). Here, we present conditions that yield large quantities of helical $M$. jannaschii $F$ ts Z tubes using the GTP analogue GMPCPP [guanylyl- $(\alpha, \beta)$ methylene-diphosphate]. These tubes are related to the $\mathrm{Ca}^{++}$-induced tubes and sheets and present an attractive hypothesis for the structure of the in vivo FtsZ polymer.

\section{Results}

Purified FtsZ1 protein from M. jannaschii has been polymerised into helical tubes under three different conditions. Condition P1 (see Materials and Methods) is the condition that has been used to obtain $\mathrm{Ca}^{++}$-induced sheets (Löwe and Amos, 1999). The protein 'precipitates' upon addition of calcium and sheets form in solution. The insoluble protein consists almost exclusively of 'cables', helical FtsZ tubes that are very hydrophobic and are difficult to observe under the electron microscope. The few acceptable pictures that we were able to obtain all produce a helical diffraction pattern. Conditions P 2 and P 3 use GM PCPP at

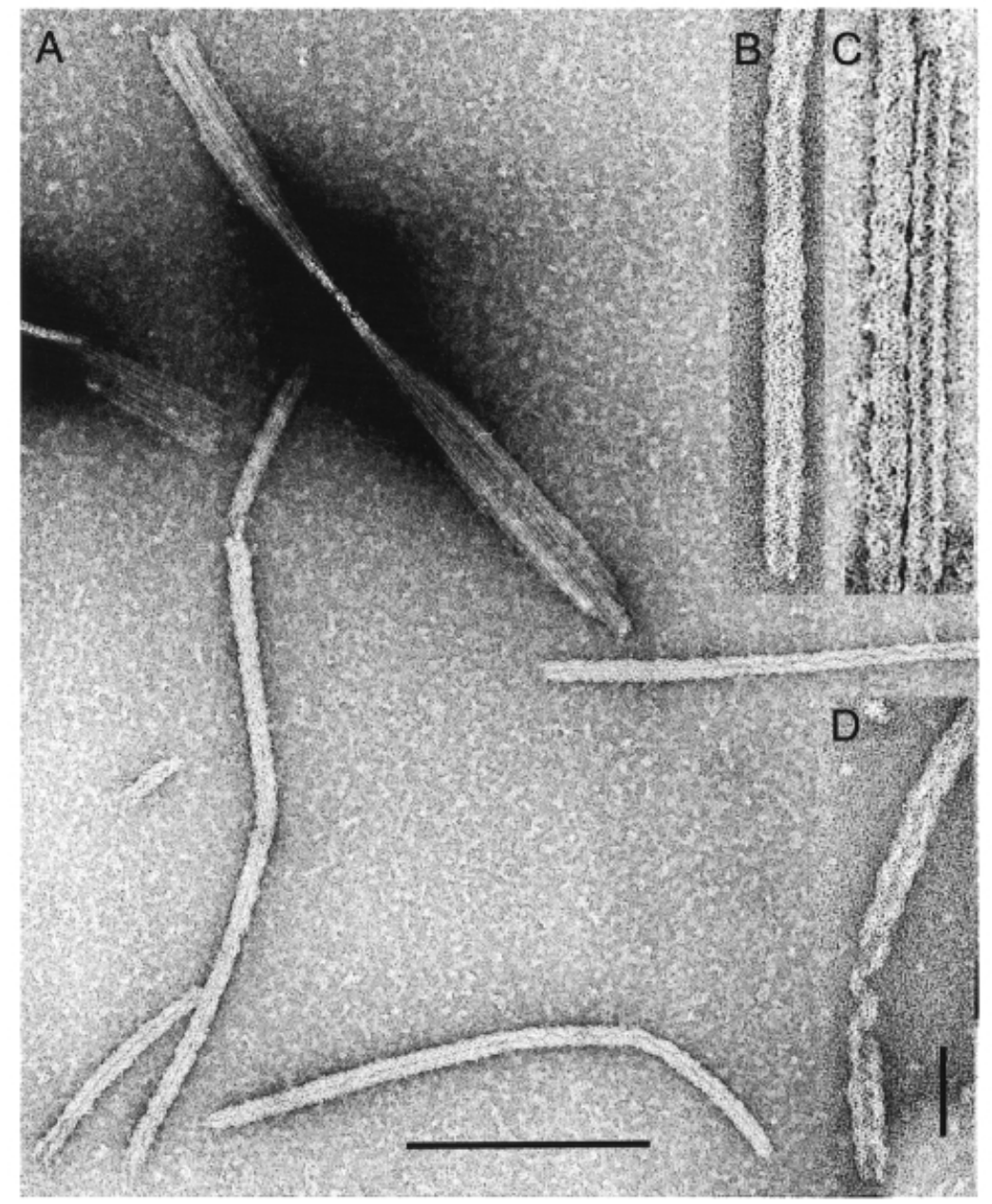

Fig. 1 FtsZ Polymers from M. jannaschii in the Presence of GMPCPP and Magnesium.

(A) Low magnification overview showing the presence of tubes and sheets on the same grid. Both forms of FtsZ polymer form with good yield and no apparent unspecific aggregation is visible. Scale bar: $500 \mathrm{~nm}$. (B) Helical FtsZ tube whose center is more heavily stained at its two ends. (C)A filled and an empty helical FtsZ tube side by side in the same preparation, polymerisation condition P2 (see Materials and Methods). (D) A partially unwound helical tube (condition P3). Scale bar for (B)- (D): $100 \mathrm{~nm}$. 
$10 \mathrm{~mm}$ and $2 \mathrm{~mm}$, respectively. Both of these conditions produce sheets and cables with high yield as shown in Figure $1 \mathrm{~A}$. Condition $\mathrm{P} 3$ produced some tubes that show obvious unwinding, as shown in Figure 1D. The unwinding demonstrates the helical nature of the tubes as they are composed of curved ribbons of protofilaments. Many of the tubes assembled under condition P3 are empty (Figures $1 C$ and $2 C$ ), whereas tubes assembled under condition $\mathrm{P} 2$ show stain exclusion everywhere, indicating that the core of the helix is filled with protein (Figure 1A, 2B). Some tubes were only partially empty, usually at either end (Figure 1B). Not many stretches of tubes were straight over long distances and most had to be straightened, as described under Materials and M ethods, in order to obtain clear diffraction patterns. Representative tubes from the three polymerisation conditions and their diffraction patterns are shown in Figure 2. Tubes assembled in the presence of calcium show layer lines at repeats of $144 \AA$ and $72 \AA$
(Figure 2D) as published earlier (Löwe and Amos, 1999). Tubes assembled in the presence of GMPCPP show repeats of $\sim 200 \AA, 100 \AA, 67 \AA$, and $50 \AA$ (Figure $2 E, F$ ). The patterns from filled and empty GM PCPP tubes are similar, suggesting that the core filaments of the filled tubes either do not contribute to the diffraction patterns or that their symmetry is related to that of the outer shell. In the case of both $\mathrm{Ca}^{++}$-induced and GMPCPP-induced tubes, all of the obvious layerlines are merely different orders of only one cell dimension of the helical surface lattice, that is, the spacing between groups of helical filaments. We have not yet identified any layerlines arising from a subunit periodicity along the filaments. We determined the number of separate filament groups from the two sets of diffraction patterns. The peaks on opposite sides of the $\mathrm{Ca}^{++}$-tube $144 \AA$ and the GMPCPP-tube $200 \AA$ layerlines were clearly out of phase, indicating an odd-order helical family, and the positions of the first peaks were consistent with a
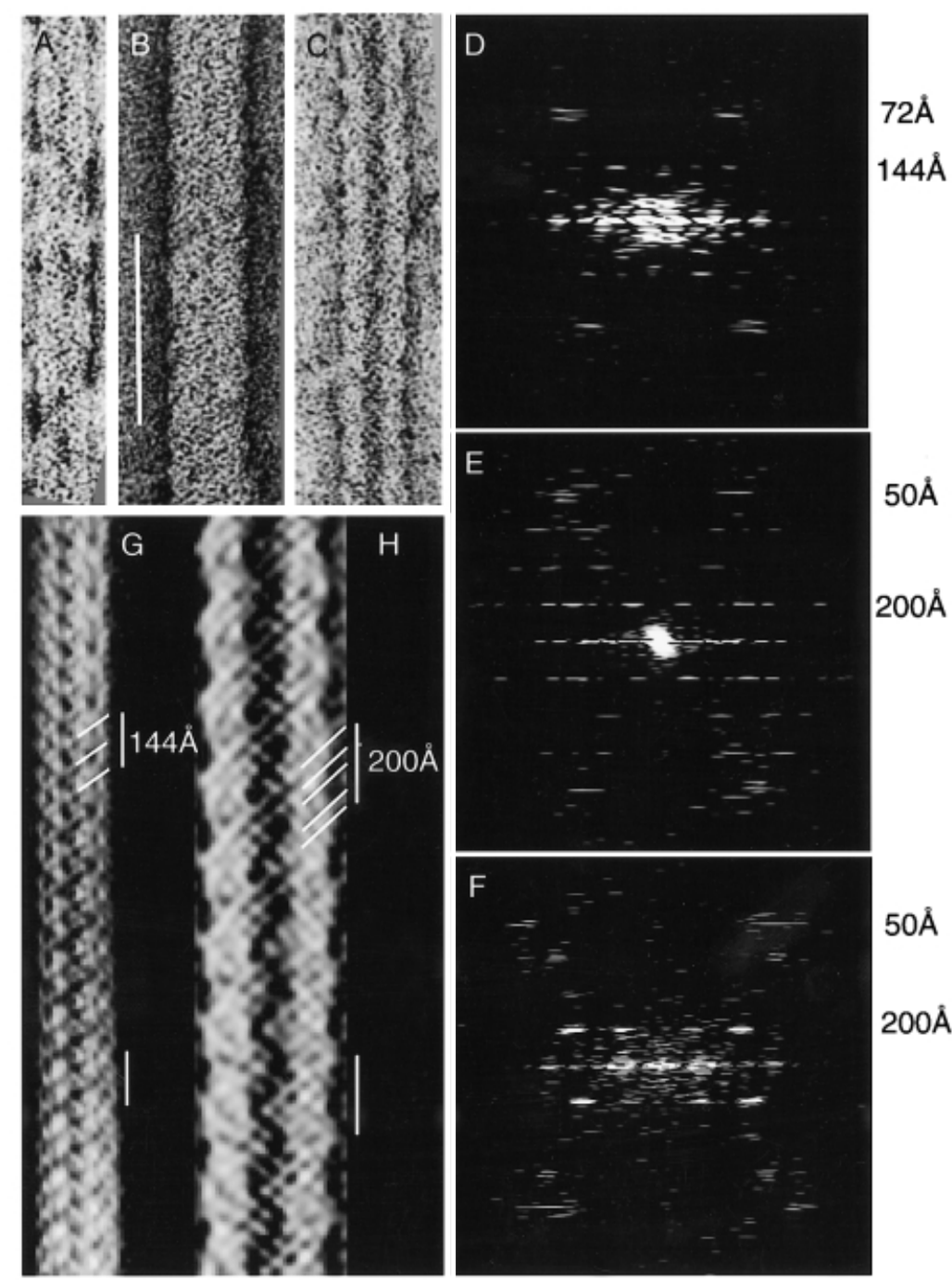

Fig. 2 Helical Tubes of FtsZ, Their Diffraction Patterns and Filtered Images.

(A, D) Ca ${ }^{++}$-GTP helical FtsZ tube and its diffraction pattern (a different example from that previously published in Löwe and Amos, 1999). Scale bar: $100 \mathrm{~nm}$. (B, E) Filled GMPCPP FtsZ tube and its diffraction pattern. (C, F) Empty GMPCPP tube and its diffraction pattern. $(G, H)$ Filtered images from images (A) and (C). The repeat length is marked alongside. In (G), a repeat includes 2 filaments, $62 \AA$ apart and running at an angle of $30^{\circ}$. In (H), four filaments at an angle of $44^{\circ}$ make up the repeat. Their sideways separations appears to be $\sim 37 \AA$ $\sim 30 \AA, \sim 43 \AA$ and $\sim 30 \AA$, but the upper two of the marked filaments are at a larger radius. 
A

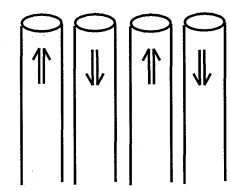

B
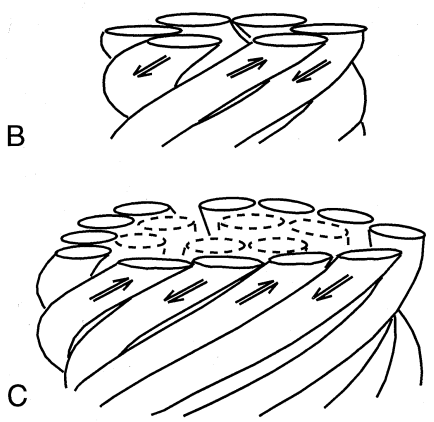

Fig. 3 Proposed Relationship between Sheets and Tubes. (A) Sheets of M. jannaschii FtsZ were shown (Löwe and Amos, 1999 ) to consist of thick filaments (parallel pairs of protofilaments). Adjacent pairs of thick filaments are anti-parallel.

(B) Each $\mathrm{Ca}^{++}$-GTP tube is made up of 6 helically-twisted thick filaments. Pairing of the thick filaments means that there is a family of three identical helical units.

(C) A GMPCPP tube consists of 3 independent groups of 4 filaments, each of which probably corresponds to a thick filament (see text). The filled tubes appear to contain additional filaments (dashed lines) but they are not sufficiently well-ordered in our specimens for their number and arrangement to be determined.

3-start family (Figure 3B). The phases and first-peak positions on the higher-order layerlines supported this assignment. Thus, the layerlines in the $\mathrm{Ca}^{++}$-tube pattern can be indexed as $(3,1)$ and $(6,2)$; those in the GMPCPP patterns can be indexed as $(3,1),(6,2),(9,3)$ and $(12,4)$. We made filtered images by masking out everything in the diffraction patterns apart from the above-mentioned layerlines and reverse Fourier transforming. Examples are shown in Figure $2 \mathrm{G}$ and $2 \mathrm{H}$. From such images it can be seen that a group of filaments producing the $144 \AA$ longitudinal spacing of the $\mathrm{Ca}^{++}$-induced tubes consists of a pair of filaments. Thus, the second layerline $(6,2)$ can be directly related to a family of $6 \sim 62 \AA$ wide filaments running in parallel around the helical axis, with a slope of $\sim 30^{\circ}$ and a pitch of $\sim 430 \AA(3 \times 144 \AA)$. In the case of the GMPCPP-induced tubes, groups producing the $\sim 200 \AA$ longitudinal spacing consist of 4 unequally-spaced filaments, with a slope of $\sim 44^{\circ}$ and a pitch of $\sim 600 \AA$. Thus, the GMPCPP tubes have a total of 12 filaments running in parallel around the axis and the fourth layerline $(12,4)$ corresponds to their average longitudinal spacing of $50 \AA$. Two of the 4 filaments tend to appear brighter than the others (see Figure $2 \mathrm{H}$ ) and lead to bulgy outlines at the sides of the tube, suggesting that they run at slightly higher radii than the darker pair, which may be masked by negative stain lying in grooves between the ridges formed by the outer pair of filaments. The distance between the resolved filaments is small, as compared with the $\mathrm{Ca}^{++}$-induced tubes. The spacing would be compatible with the broadest width of individual protofilaments. However, the empty tubes are too thick $(\sim 100 \AA)$ to be made up of one layer of protofilaments. It therefore seems likely that the GMPCPP tubes also consist of thick filaments like those in the sheets and the $\mathrm{Ca}^{++}$ GTP tubes. The distances between neighbouring filaments probably appear foreshortened because of their slightly different radial positions. Very low resolution 3D re-
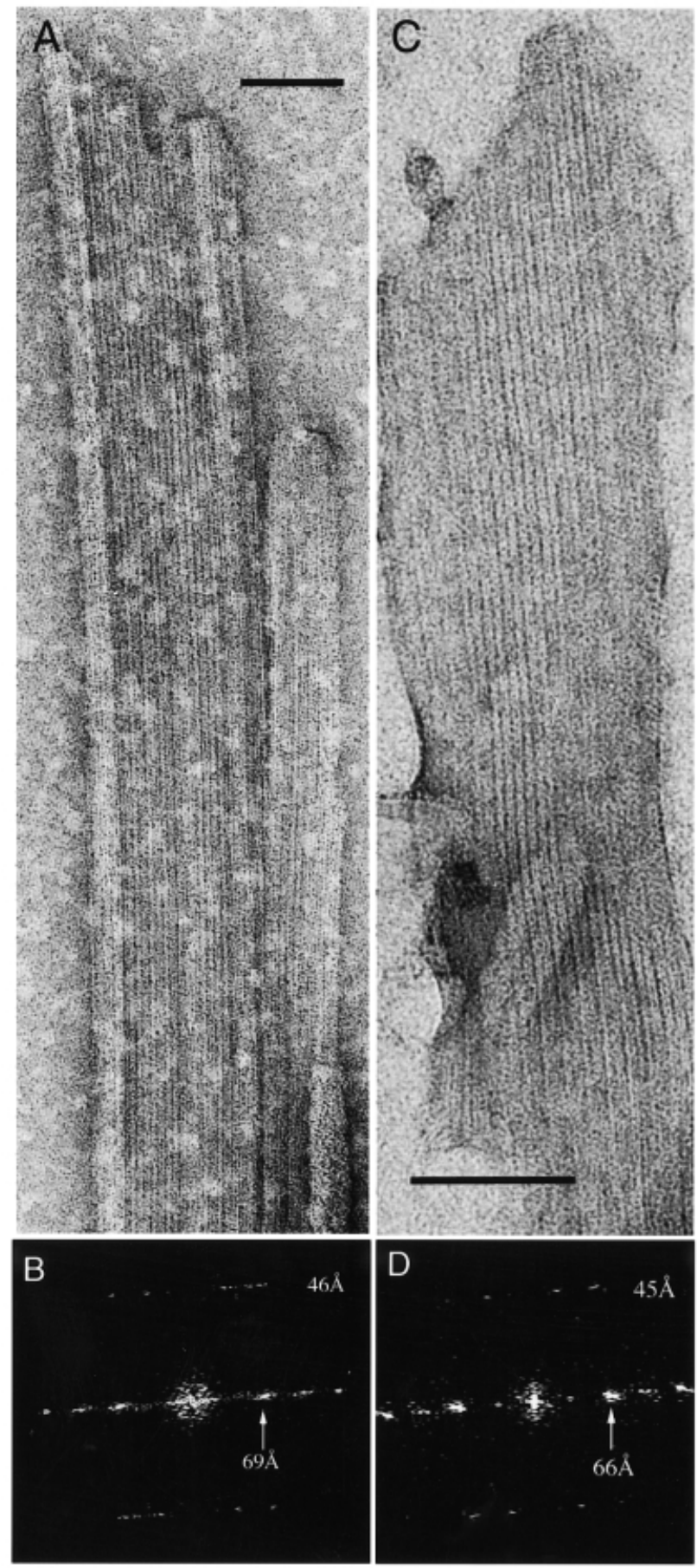

Fig. 4 Sheets of FtsZ from M. jannaschii Grown in the Presence of GMPCPP.

(A, C) FtsZ sheets assembled under condition P2. The left-hand edge of $(A)$ consists of a roll of straight filaments. $(B, D)$ Diffraction patterns from parts of $(A)$ and $(C)$. S pacings marked alongside the diffraction patterns are based on the nominal magnification. Scale bars: $100 \mathrm{~nm}$. 
constructions (sketched in Figure 3C) provided support for this interpretation of the filament arrangement.

Diffraction patterns from flat pieces of sheets assembled in the presence of GMPCPP (Figure 4) are closely similar to patterns obtained earlier from $\mathrm{Ca}^{++}$-induced sheets (Löwe and Amos, 1999). These sheets also appear to consist of antiparallel pairs of thick filaments with a longitudinal subunit repeat of $43-46 \AA$ and a filament separation of $65-70 \AA$. The only apparent difference is whereas $\mathrm{Ca}^{++}$-induced sheets were often twisted in the form of taut ribbons, GMPCPP-induced sheets appeared less tense and sometimes became rolled up at their edges (Figure 4A) or compressed sideways.

\section{Discussion}

The finding that FtsZ, the bacterial tubulin homologue, forms a ring around the invaginating septum prompted the intuitive hypothesis that FtsZ forms a polymer during septation (Bi and Lutkenhaus, 1991; Lutkenhaus, 1993). Although no direct evidence for FtsZ involvement in force generation has been obtained so far, it is generally thought that force is generated either by motor proteins moving on FtsZ tracks or by FtsZ polymer dynamics analogous to microtubule or $\mathrm{F}$-actin function. It is clear that FtsZ forms tubulin-like protofilaments (Erickson, 1995; Löwe and Amos, 1999) and that FtsZ plays a very direct role in the placement of additional components of the septum, as indicated by an insertion mutant that assembles spirals instead of rings (Bi and Lutkenhaus, 1992; Addinall and Lutkenhaus, 1996). Unfortunately, ultrastructural information about the septum and the in vivo $\mathrm{FtsZ}$ polymer has not been obtained so far. In the absence of knowledge about FtsZ's structure in the Z-ring we can only try to find in vitro conditions for FtsZ polymerisation that have the following characteristics: the polymerisation conditions should be universal and work for most FtsZ proteins with the same resulting polymer. The condition should yield an ordered polymer that does not form an irregular network. Furthermore, the functional polymer should be formed from only 10 - 20 protofilaments or less: the estimate of $15000 \mathrm{FtsZ}$ molecules per cell (Dai and Lutkenhaus, 1992; Lu et al., 1998) would translate into about $60-70 \mu \mathrm{m}$ in length if all molecules assemble into protofilaments. For a septum of $1 \mu \mathrm{m}$ diameter, this would give 10 - 20 protofilaments in the Z-ring, if FtsZ forms a continuous polymer. Each polymer would have fewer protofilaments if the Z-ring is made up of discontinuous and overlapping polymers, as in the eukaryotic cytokinetic actin structure which performs a similar, if not identical, function to the Z-ring in bacterial cell division. FtsZ has been polymerised in vitro to form curved thin filaments, sheets and protofilament bundles. None of these polymer forms seem to be suitable for FtsZ's role in the cell. When we investigated the $\mathrm{Ca}^{++}$-induced formation of sheets of FtsZ we detected helical tubes but were not able to produce enough acceptable pictures for a detailed investigation. Two recent reports
Trusca etal., 1998; Lu et al., 2000) presented similar helical tubes using FtsZ from E. coli and DEAE dextran/GDP. In our opinion, the helical Fts $Z$ tubes represent the most likely hypothesis presented so far for the in vivo FtsZ polymer. Although we do not know which of the three forms of tube we have assembled is most likely to occur in a cell, each type consists of a defined and small number of protofilaments per length unit, is flexible enough to bend around the constricting septum and does not stick together to form a network of filaments. The last requirement mentioned above, a polymerisation condition that is universal or at least transferable from one protein to the other is not fulfilled, as M. jannaschii FtsZ does not form tubes under the conditions reported for E. coli FtsZ (Trusca et al., 1998; Lu et al., 2000; data not shown). This, however, may not be very surprising as $M$. jannaschii is a hyperthermophilic archaeon and grows above $80^{\circ} \mathrm{C}$. It is surprising to find that we can produce helical tubes with $M$. jannaschii Fts $Z$ using the supposedly non-hydrolysable GTP analogue GMPCPP, as FtsZ is thought to form straight filaments when bound to GTP and curved protofilaments when bound to GDP (Lu and Erickson, 1997; Lu et al., 2000). Clearly, helical FtsZ tubes are made from curved filaments. When GMPCPP is replaced with GDP or GTP in our experiments, no polymer is formed orbundles of straight filaments form. One explanation for the formation of curved filaments in the presence of GMPCPP is slow hydrolysis, which we can observe when crystals of Fts $Z$ are soaked with GMPCPP and left for prolonged periods of time (not shown). GMPCPP has been found to be hydrolysed by tubulin, although much more slowly than GTP (Hyman et al., 1992), but its hydrolysis by FtsZ has not yet been investigated. In solution, GMPCPP hydrolysis by FtsZ is expected to be much faster than in a crystal because of assembly-dependent GTPase activation. GTP may not induce helical tubes of $M$. jannaschii Fts Z because protofilament extension under our in vitro conditions may be too fast for the formation of ordered polymers. DEAE dextran, which could substitute for an accessory protein in aiding the assembly of $E$. coli FtsZ, seems not to have any influence on the $M$. jannaschii FtsZ polymerisation process (data not shown).

We have investigated helical tubes formed under two different conditions. Insoluble tubes formed in the presence of calcium while more soluble tubes formed reliably in the presence of GMPCPP. Analysis of diffraction patterns and filtered images of these tubes allowed us to determine their filament substructure. Both tubes are 3-start helices and differ mainly in the number of subfilaments. The calcium-induced tubes contain 2 subfilaments in a group, whereas the GMPCPP tubes contain 4 subfilaments, giving 6 and 12 subfilaments pertube. The spacing orthogonal to the subfilament direction, inclined by $30-45^{\circ}$ from the tube axis, is $\sim 60 \AA$ for the calcium tubes. It is less for the unequally-spaced subfilaments of the GMPCPP tubes but their overall separation, including the radial differences, is probably similar. Since both types of helical FtsZ tubes have been obtained under conditions that form sheets as well, we compared the sheets obtained in the 
presence of GMPCPP with the $\mathrm{Ca}^{++}$-induced sheets (Löwe and Amos, 1999) and found them to have very similar lattice constants and diffraction patterns. We therefore conclude that the GMPCPP sheets contain alternating antiparallel thick filaments, each composed of two parallel FtsZ protofilaments, like the $\mathrm{Ca}^{++}$-induced sheets. A spacing of $50-60 \AA$ in the tubes, which has been seen in the $E$. coli helical FtsZ tubes as well (Lu et al., 2000), could be generated by a double protofilament or a single protofilament. In an atomic model of the FtsZ protofilament, the thinnest diameter is $25 \AA$ and the thickest $50 \AA$, excluding helix HO (Löwe and Amos, 1998, 1999). We tend to think that the M. jannaschii FtsZ tubes are composed of double protofilaments as well, since they form under the same conditions as the sheets (see Figure 1), and that the tube and sheet polymer forms may represent the parallel and antiparallel subfilament arrangement. The nature of the subfilaments could be investigated using cryo-EM and higher resolution three-dimensional image reconstruction.

Polymerisation condition P2 yielded filled tubes, whereas condition P3 yielded empty or partially filled tubes. We were not able to determine what the core consists of but it may be another protein structure following the helical symmetry.

The helical FtsZ tubes found in this study and by others are very different from normal eukaryotic microtubules. $M$ icrotubules contain straight or almost straight protofilaments formed with $\beta$-tubulin in the GTP state, depending of the number of protofilaments (Wade et al., 1990). After hydrolysis of GTP to GDP, the relaxed protofilament would prefer to adopt a curved conformation, thus giving dynamic instability to the microtubule (Desai and Mitchison, 1997). This mechanism stores energy in the microtubule lattice during tube formation so that an additional source of energy is not essential for disassembly (Mitchison and Kirschner, 1984). Since FtsZ tubes appear to contain significantly curved filaments, this polymermay not be dynamic and external factors may be required to disassemble the tubes. Here the role of GTP hydrolysis could be that of a lock, making disassembly unlikely. We think it is possible that GTP hydrolysis actually promotes lateral interactions between FtsZ protofilaments. FtsZ lacks the M-loop of tubulin (Nogales et al., 1999) that is responsible for the most important lateral interaction between tubulin protofilaments, so lateral interactions between FtsZ protofilaments may well be quite different from those in microtubules. Mitchison and Kirschner (1984) suggested that dynamic instability of microtubules was important in ensuring that microtubules assembled without proper initiation would automatically soon disassemble. The dynamic property of Fts Z protofilaments is likely to serve a similar purpose. As mentioned above, it seems to be necessary for practically all of the molecules present in the cell to assemble in a specific region. The average concentration of Fts Z throughout the cell may be insufficient for unassisted assembly into tubes although it is probably above the critical concentration of 20 to $50 \mu \mathrm{g} / \mathrm{ml}$ for polymerisation into smaller structures (Lu et al., 1998; M ukherjee and Lutkenhaus, 1998; Mukherjee and Lutkenhaus, 1999). Thus, it should only be necessary for individual protofilaments and small bundles to be dynamically unstable.

Microtubules are the tracks of motor proteins that move along protofilaments to produce linear force (Hirokawa, 1998). A helical tube does not seem to be optimal for this task due to topological implications, although it could rotate if neither end is fixed and the motors are involved in holding it on to the membrane.

To observe the subunit repeat along the protofilaments it will be necessary to use cryo-EM to obtain higher resolution images and to obtain less bent polymers. Three-dimensional reconstruction of ice-embedded helical FtsZ polymers will also allow for investigation of FtsZ:protein complexes with components of the septum interacting with FtsZ like FtsA, ZipA, FtsQ, and others. The helical tubes may also be very useful for setting up motility assays under the DIC light microscope with FtsZ filaments and bacterial lysates or purified proteins, to search for bacterial motor proteins. Prelimary experiments indicate that they are large enough to be visible by light microscopy.

The helical FtsZ cables presented in this study form with high yield. In addition to their potential as important tools for the investigation of FtsZ:protein complexes with cryoEM and video-enhanced light microscopy, they provide a promising hypothesis for the in vivo polymer of FtsZ.

\section{Materials and Methods}

FtsZ1 from Methanococcus jannaschii was expressed and purified as described before (Löwe, 1998). The protein was stored at $4{ }^{\circ} \mathrm{C}$ in $20 \mathrm{~mm}$ TRIS, $1 \mathrm{~mm}$ EDTA, $1 \mathrm{~mm} \mathrm{Na}$-azide. Nucleotides were used as neutralised $100 \mathrm{~mm}$ stock solutions ( $\mathrm{pH}$ 7.0). GMPCPP was synthesised as described by Hyman et al. (1992). For FtsZ tube formation, three different polymerisation conditions were used: P1 (as previously published in Löwe and Amos, 1999): $8 \mathrm{~mm}$ magnesium acetate, $8 \mathrm{~mm}$ calcium chloride and $8 \mathrm{~mm}$ GTP were added to Fts Z1 to give a final protein concentration of $2 \mathrm{mg} / \mathrm{ml}$. The cloudy reaction product was vortexed for $10 \mathrm{~s}$. Polymerisation condition P2: $10 \mathrm{~mm} \mathrm{GMPCPP}$ and $20 \mathrm{~mm}$ magnesium acetate were mixed with FtsZ1 to give a final protein concentration of $0.8 \mathrm{mg} / \mathrm{ml}$. P2 yields sheets and filled tubes. P 3: as P2 but with $2 \mathrm{mM}$ GMPCPP and $4 \mathrm{~mm}$ magnesium acetate. P 3 yields exclusively empty tubes. All reactions were incubated for $20 \mathrm{~min}$ at $37^{\circ} \mathrm{C}$. Samples were negatively stained with $1 \%$ uranyl acetate and observed in a Philips $20880 \mathrm{kV}$ electron microscope. Electron micrographs used for image processing were photographed at magnifications between 25000 and 63000 . They were scanned with a Zeiss SCAI scanner at 28 or 14 micrometer step size and processed using the MRC package of programs (C rowther et al., 1996). After spline-fitting a line down the axis of each image of a curved polymer, unbending was achieved by bilinear interpolation (Egelman, 1986). The straightened image was boxed and floated and its Fourier transform $(512 \times 1024$ or $512 \times 512$, depending on the length of polymer available) was calculated. Filtered images were obtained by masking out unwanted regions of the Fourier transform and calculating a reverse Fourier transformation. For a more detailed analysis, complex data along each layerline were extracted and the phases on opposite sides 
of the meridian were compared. For each possible indexing scheme, a search was made over a range of $x$-shifts and tilt angles (out of the plane normal to the direction of view) to find values that would give an overall minimum for the amplitude-weighted phase differences (or differences- $\pi$, in the case of odd-order helical families) at equal distances from the meridian. The values of the overall minima provided an indication of the most likely indexing scheme. After appropriate correction of the phases, each Fourier transform yielded 2 datasets, from the 'near' and 'far' sides of the image. The phases in each dataset were then compared to estimate the z-shift and rotation around the helix axis required to bring each particle into conjunction with a chosen reference. The shifts that minimised the weighted difference in the phases were found by searching values that covered a complete repeat. Agreement between the near and far sides of a particular image was used as an indication of the quality of the data. The phases in each dataset were corrected to shift the structure into the reference orientation and an average of the datasets was used to reconstruct 3D images for comparison with the recorded electron micrographs and filtered images.

\section{Acknowledgements}

This work was carried out with some support from the Human Frontier Science Program. We thank Dr. T. J . Mitchison (Harvard Medical School) for advice about GMPCPP and are grateful to Dr. N. Caplan and Professor G. M. Blackburn (Department of Chemistry, University of Sheffield) who synthesised this nucleotide for us.

\section{References}

Addinall, S.G., and Lutkenhaus, J . (1996). FtsZ-spirals and -arcs determine the shape of the invaginating septa in some mutants of Escherichia coli. Mol. Microbiol. 22, 231 - 237.

Bi, E., and Lutkenhaus, J . (1991). Ftsz ring structure associated with division in Escherichia coli. Nature 354, 161 - 164.

Bi, E., and Lutkenhaus, J . (1992). Isolation and characterization of FtsZ alleles that affect septal morphology. J. Bacteriol. 174, 5414 - 5423.

Bramhill, D. (1997). Bacterial cell division. Annu. Rev. Cell Dev. Biol. 13, $395-424$.

Bramhill, D., and Thompson, C.M. (1994). GTP-dependent polymerization of Escherichia coli FtsZ protein to form tubules. Proc. Natl. Acad. Sci. USA 91, 5813 - 5817.

Crowther, R.A., Henderson, R., and Smith, J. M. (1996). MRC image processing programs. J . Struct. Biol. 116, 9 - 16.

Dai, K., and Lutkenhaus, J . (1992). The proper ratio of FtsZ to FtsA is required for cell division to occur in Escherichia coli. J . Bacteriol. 174, 6145 - 6151.

Desai, A., and Mitchison, T.J . (1997). Microtubule polymerization dynamics. Annu. Rev. Cell Dev. Biol. 13, 83 - 117.

Donachie, W.D. (1993). The cell-cycle of Escherichia coli. Annu. Rev. Microbiol. 47, 199 - 230.

Egelman, E.H. (1986). An algorithm for straightening images of curved filamentous structures. Ultramicroscopy 19, 367 - 373

Erickson, H.P. (1995). FtsZ, a prokaryotic homolog of tubulin? Cell $80,367-370$.

Erickson, H. P. (1998). Atomic structures of tubulin and FtsZ. Trends Cell Biol. 8, 133-137.

Erickson, H.P., and Stoffler, D. (1996). Protofilaments and rings, 2 conformations of the tubulin family conserved from bacterial Ftsz to alpha/beta and gamma-tubulin. J . Cell Biol. 135, 5 - 8.
Erickson, H.P., Taylor, D.W., Taylor, K.A., and Bramhill, D. (1996). Bacterial cell division protein $\mathrm{FtsZ}$ assembles into protofilament sheets and minirings, structural homologs of tubulin polymers. Proc. Natl. Acad. Sci. USA 93, 519 - 523.

Hirokawa, N. (1998). Kinesin and dynein superfamily proteins and the mechanism of organelle transport. Science 279, 519 - 526.

Hyman, A.A., Salser, S., Drechsel, D.N., Unwin, N., and Mitchison, T.J . (1992). Role of GTP hydrolysis in microtubule dynamics: information from a slowly hydrolyzable analogue, GMPCPP. Mol Biol Cell 3, 1155 - 1167.

Löwe, J. (1998). Crystal structure determination of FtsZ from Methanococcus jannaschii. J . Struct. Biol. 124, 235 - 243.

Löwe, J ., and Amos, L.A. (1998). Crystal structure of the bacterial cell-division protein FtsZ. Nature 391, 203 - 206.

Löwe, J ., and Amos, L.A. (1999). Tubulin-like protofilaments in $\mathrm{Ca}^{2+}$-induced FtsZ sheets. EMBO J . 18, 2364 - 2371.

Lu, C.L., and Erickson, H.P. (1999). The straight and curved conformation of FtsZ protofilaments - evidence for rapid exchange of GTP into the curved protofilament. Cell Struct. Funct. 24, $285-290$.

Lu, C.L., Stricker, J ., and Erickson, H.P. (1998). FtsZ from Escherichia coli, Azotobacter vinelandii, and Thermotoga maritima - quantitation, GTP hydrolysis, and assembly. Cell Mot. Cytoskel. $40,71-86$.

Lu, C.L., Reedy, M., and Erickson, H.P. (2000). Straight and curved conformations of Fts $Z$ are regulated by GTP hydrolysis. J . Bacteriol. 182, $164-170$.

Lutkenhaus, J. (1993). FtsZ ring in bacterial cytokinesis. Mol. Microbiol. 9, 403 - 409.

Lutkenhaus, J., and Addinall, S.G. (1997). Bacterial cell division and the $Z$ ring. Annu. Rev. Biochem 66, 93 - 116.

Mitchison, T.J ., and Kirschner, M. W. (1984). Dynamic instability of microtubule growth. Nature 312, 237 - 242.

Mukherjee, A., and Lutkenhaus, J . (1994). Guanine nucleotidedependent assembly of FtsZ into filaments. J . Bacteriol. 176, 2754 - 2758.

Mukherjee, A., and Lutkenhaus, J . (1998). Dynamic assembly of FtsZ regulated by GTP hydrolysis. EMBO J . 17, 462 - 469.

Mukherjee, A., and Lutkenhaus, J . (1999). Analysis of FtsZ assembly by light scattering and determination of the role of divalent metal cations. J. Bacteriol. 181, $823-832$.

Nogales, E., Downing, K.H., Amos, L.A., and Löwe, J . (1998a). Tubulin and FtsZ form a distinct family of GTPases. Nature Struct. Biol. 5, $451-458$

Nogales, E., Wolf, S.G., and Downing, K.H. (1998b). Structure of the $\alpha / \beta$-tubulin dimer by electron crystallography. Nature 391 , $199-203$.

Nogales, E., Whittaker, M., Milligan, R.A., and Downing, K.H. (1999). High-resolution model of the microtubule. Cell 96, $79-$ 88.

Rothfield, L., J ustice, S., and GarciaLara, J . (1999). Bacterial cell division. Annu. Rev. Gen. 33, 423 - 448.

Trusca, D., Scott, S., Thompson, C., and Bramhill, D. (1998). Bacterial SOS checkpoint protein SulA inhibits polymerization of purified FtsZ cell division protein. J. Bacteriol. 180, 3946 3953.

Wade, R.H., Chrétien, D., and J ob, D. (1990). Characterization of microtubule protofilament numbers. How does the surface lattice accommodate? J . Mol. Biol. 212, 775 - 786.

Yu, X.C., and Margolin, W. (1997). Ca ${ }^{2+}$-mediated GTP-dependent dynamic assembly of bacterial cell division protein FtsZ into asters and polymer networks in vitro. EMBO J . 16, 5455 5463.

Received May 24, 2000; accepted J uly 14, 2000 